\title{
Development and validation of the fracture risk scale home care (FRS-HC) that predicts one-year incident fracture: an electronic record-linked longitudinal cohort study
}

Caitlin McArthur ${ }^{1,2^{*}}$ D, George Ioannidis ${ }^{1,2}$, Micaela Jantzi ${ }^{3}$, Jonathon D. Adachi ${ }^{1}$, Lora Giangregorio ${ }^{3,4}$, John Hirdes ${ }^{3}$ and Alexandra Papaioannou ${ }^{1,2}$

\begin{abstract}
Background: Fractures have dire consequences including pain, immobility, and death. People receiving home care are at higher risk for fractures than the general population. Yet, current fracture risk assessment tools require additional testing and assume a 10-year survival rate, when many die within one year. Our objectives were to develop and validate a scale that predicts one-year incident hip fracture using the home care resident assessment instrument (RAl-HC).

Methods: This is a retrospective cohort study of linked population data. People receiving home care in Ontario, Canada between April 1st, 2011 and March 31st, 2015 were included. Clinical data were obtained from the RAI-HC which was linked to the Discharge Abstract Database and National Ambulatory Care Reporting System to capture one-year incident hip fractures. Seventy-five percent $(n=238,011)$ of the sample were randomly assigned to a derivation and $25 \%(n=79$, 610) to a validation sample. A decision tree was created with the derivation sample using known fracture risk factors. The final nodes of the decision tree were collapsed into 8 risk levels and logistic regression was performed to determine odds of having a fracture for each level. c-Statistics were calculated to compare the discriminative properties of the full, derivation, and validation samples.
\end{abstract}

Results: Approximately $60 \%$ of the sample were women and $53 \%$ were 80 years and older. A total of 11,526 (3.6\%) fractures were captured over the 1-year time period. Of these, 5057 (43.9\%) were hip fractures. The proportion who experienced a hip fracture in the next year ranged from $0.3 \%$ in the lowest risk level to $5.2 \%$ in the highest risk level. People in the highest risk level had 18.8 times higher odds (95\% confidence interval, 14.6 to 24.3) of experiencing a hip fracture within one year than those in the lowest. c-Statistics were similar for the full (0.658), derivation (0.662), and validation (0.645) samples.

Conclusions: The FRS-HC predicts hip fracture over one year and should be used to guide clinical care planning for home care recipients at high risk for fracture. Our next steps are to develop a fracture risk clinical assessment protocol to link treatment recommendations with identified fracture risk.

Keywords: Fractures, Home care, osteoporosis, older adults, interRAl, Cohort

\footnotetext{
* Correspondence: cmcarthu@uwaterloo.ca

'McMaster University, 1200 Main Street, Hamilton, Ontario L8S 4L 8, Canada ${ }^{2}$ GERAS Centre for Aging Research, 88 Maplewood Avenue, 88 Maplewood Avenue, Hamilton, Ontario L8M 1W9, Canada

Full list of author information is available at the end of the article
}

C C The Author(s). 2020 Open Access This article is licensed under a Creative Commons Attribution 4.0 International License, which permits use, sharing, adaptation, distribution and reproduction in any medium or format, as long as you give appropriate credit to the original author(s) and the source, provide a link to the Creative Commons licence, and indicate if changes were made. The images or other third party material in this article are included in the article's Creative Commons licence, unless indicated otherwise in a credit line to the material. If material is not included in the article's Creative Commons licence and your intended use is not permitted by statutory regulation or exceeds the permitted use, you will need to obtain permission directly from the copyright holder. To view a copy of this licence, visit http://creativecommons.org/licenses/by/4.0/. The Creative Commons Public Domain Dedication waiver (http://creativecommons.org/publicdomain/zero/1.0/) applies to the data made available in this article, unless otherwise stated in a credit line to the data. 


\section{Background}

Worldwide, there has been a shift from institutional models of care (i.e., long-term care) to supporting older adults in their own homes. The consequence of the shift is that an increasing number of frail, medically-complex older adults are reliant on support from service such as home care which is defined as receiving nursing or professional services (e.g., physical or occupational therapy) for 60 days or more within a person's home [1]. Because they are more medically complex, people receiving home care experience a higher incidence of negative events such as falls and fractures than the average population [2], further increasing their risk of functional dependence, institutionalization and mortality. The incidence of hip fracture for people receiving home care is high, at 24.4 per 1000 person-years [3] compared to 5.7 per 1000 person-years in the average population [4]. Fractures pose a significant burden to the health, quality of life, and mortality of older adults receiving home care services [3]. After a hip fracture, $25 \%$ of people require institutionalization [5], and over $20 \%$ will die [6]. Hip fractures also carry a significant economic burden; the costs associated with fractures in home care in Canada are \$274 million [7]. Home care clients at risk for fractures must be identified and strategies must be implemented to prevent the loss of mobility and independence, and increased risk of death.

Fracture risk is commonly identified through risk assessment tools, such as the FRAX [8]. However, current fracture assessment tools may not be valid or generalizable for some medically complex home care recipients. First, current risk assessment tools do not capture potential risk factors that may be more relevant for assessing risk among the home care population (e.g., cognitive impairment, multi-morbidity and falls risk). Fracture risk outputs may not provide accurate estimates for home care recipients with multiple comorbidities. Current assessment tools rely on data often unavailable in routine home care assessments, such as bone mineral density, adding to the assessment burden of this sector. Finally, imminent fracture risk (i.e., within the next year) must be the target for fracture risk assessment with the vulnerable home care population. As many as $17 \%$ of home care clients die within one year from admission [2]. Risk estimates with a longer prediction timeframe will underestimate imminent fracture risk and lack the urgency for prevention strategies to be implemented.

The Resident Assessment Instrument - Home Care (RAI-HC) [9] is a comprehensive, standardized tool implemented as part of routine clinical practice across several Canadian provinces. The RAI-HC is completed upon admission into a long-stay home care programs and includes person-level data elements and outcome scores [9]. Outputs from the RAI-HC can be used to guide practice and identify home care recipients at risk for negative events or outcomes, such as fractures. The RAI-HC is routinely collected for all long-stay home care recipients in Canada and internationally meaning that fracture risk identification could be automatically incorporated into daily practice without additional documentation burden. Our objectives were to develop and validate a scale that predicts one-year incident hip fracture using the home care resident assessment instrument (RAI-HC). Our team has previously developed a Fracture Risk Scale for long-term care using a similar assessment system (MDS 2.0) [10]. However, given the observed difference in fracture incidence [11] and population characteristics [12] between home and long-term care, we considered it important to develop a tool specific to the unique attributes of home care recipients. Further, there continues to be large care gap for people identified at high fracture risk [13] despite bone mineral density often being available in primary care.

\section{Methods \\ Study design}

This is a retrospective cohort study of linked population data. People receiving home care in Ontario, Canada between April 1st, 2011 and March 31st, 2015 were included; home care recipients identified as end of life or receiving hospice care were excluded. The final sample size included 317,621 home care recipients. Seventy-five percent $(n=238,011)$ of the home care recipients were randomly assigned to a derivation and $25 \%(n=79,610)$ to a validation sample (Fig. 1). Home care recipients were classified as experiencing or not experiencing an incident hip fracture over the 1-year follow-up period. We chose hip fracture as our target variable as they are the most common type of fracture that come to clinical attention [11], the negative sequelae of hip fractures often outweigh those of other types of fractures, other prediction models often use hip fractures as the target outcome, and to be consistent with our previously developed Fracture Risk Scale for long-term care [10].

\section{Data sources}

Clinical data were obtained from the RAI-HC. The RAI$\mathrm{HC}$ is a valid and reliable [14] standardized assessment that is completed upon admission for all recipients into long-stay home care programs in Ontario, Canada. The RAI-HC is completed by trained assessors who gather information from recipients, their family members and health care providers, and through chart review. It includes individual data elements and outcomes scores on over 200 health and social characteristics. RAI-HC data were linked to the Discharge Abstract Database (DAD) and National Ambulatory Care Reporting System (NACRS) to capture incident hip fracture. The DAD 


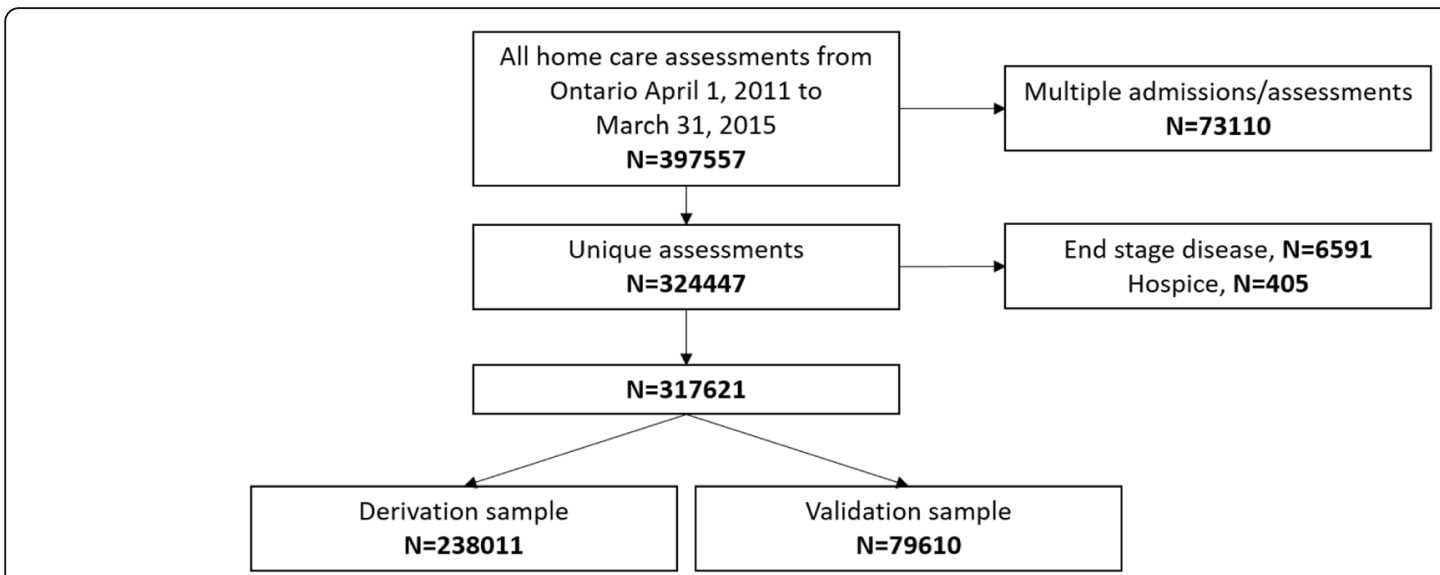

Fig. 1 Study sample flow diagram

captures inpatient hospital stay data while the NACRS captures emergency department visits $[15,16]$. Incident fracture was captured based on the definition of the Public Health Agency of Canada in the Revised Framework for National Surveillance on Osteoporosis and Osteoporosis-Related Fractures [17]. Using International Classification of Disease 10 codes, home care recipients were coded as having a fractures within 1-year of their admission assessment [hip (S72.0, S72.1 S72.2), spine (S22.0, S22.1 S 2.0, S32.8), humerus (S42.2), forearm (S52.x, S62.x), and pelvis (S32.1, S32.2, S32.4, S32.5, S32.8)]. Our target outcome was the first fracture diagnosis within one year and we did not include multiple admissions or discharges for the same diagnoses.

\section{Statistical analyses}

Clinical characteristics were expressed in count and per cent for categorical variables, and we modelled the univariate associations (odds ratio and 95\% confidence interval) between the final included risk factors and hip fracture incidence via multiple logistic regression models. We created a decision tree with the $75 \%$ derivation sample to predict incident hip fracture within one year of admission to home care using known fracture risk factors identified by previous literature $[3,10]$ and expert opinion. Decision trees are advantageous over standard additive models of risk because they allow for detection of complex interactions, identification of small but important groups at higher risk for the outcome, and inclusion of non-linear relationships $[18,19]$. One year was chosen as the target time frame given the high one-year mortality rate in home care [2] and to be consistent with our previously developed Fracture Risk Scale for long-term care [10]. Items and scales were retained based on significant association with incident hip fractures, relevance of the items, and face validity as determined by a clinical expert panel. In SAS Enterprise
Miner V.13.1 (SAS Institute), $x^{2}$ Automatic Interaction Detection used recursive partitioning to create the decision tree [20]. The final decision tree was validated at a meeting with clinical experts. Leaves of the final decision tree were combined to create the final risk levels of the scale based on examination by the expert panel of 1) the risk profiles; 2) incidence of hip fracture; and 3) proportion of the population in each leaf and risk level. An interactive decision tree analysis approach including clinical expert insight guided by statistical evidence was chosen to ensure the final tool had clinical relevance and would be applicable.

The final nodes of the decision tree were collapsed into eight risk levels and logistic regression was performed to determine the odds of having a fracture within one year of admission to home care for each level. c-Statistics were calculated to compare the discriminative properties of the full, derivation, and validation samples. All statistical analyses were completed in in SAS V9.3 (SAS Institute).

\section{Results}

The population characteristics of the derivation, validation, and combined dataset are in Table 1 . In the combined sample, $60 \%$ of the population were female and $53 \%$ were over the age of 80 years. There were 11,526 (3.6\%) incident fractures within one year from admission to home care, of which 5057 (43.9\%) were hip and 6959 (56.1\%) were other locations (wrist, spine, humerus, pelvis). Population characteristics and fracture incidence were similar across the derivation, validation, and full sample datasets. Univariate odds ratios for the final included risk factors are found in Table 2.

\section{Decision tree model}

The final decision tree model had 16 leaves (Fig. 2) that were collapsed into the eight risk levels of the Fracture 
Table 1 Characteristics of the full, derivation, and validation samples

\begin{tabular}{|c|c|c|c|}
\hline \multirow[t]{2}{*}{ Characteristic: } & \multirow{2}{*}{$\begin{array}{l}\text { Full sample } \\
N=317,621 \\
N(\%)\end{array}$} & \multirow{2}{*}{$\begin{array}{l}\text { Derivation sample } \\
N=238,011 \\
N(\%)\end{array}$} & \multirow{2}{*}{$\begin{array}{l}\text { Validation sample } \\
N=79,610 \\
N(\%)\end{array}$} \\
\hline & & & \\
\hline New fractures within one year from initial assessment & $11,526(3.6)$ & $8679(3.7)$ & $2847(3.6)$ \\
\hline Hip & $5057(1.6)$ & $3822(1.6)$ & $1235(1.6)$ \\
\hline Other fracture (spine, pelvis, humerus, wrist) & $6959(2.2)$ & $5228(2.2)$ & $1731(2.2)$ \\
\hline \multicolumn{4}{|l|}{ Age group } \\
\hline 18 to 50 & $14,354(4.5)$ & $10,693(4.5)$ & $3661(4.6)$ \\
\hline 50 to 64 & $37,652(11.9)$ & 28,297 (11.9) & $9355(11.8)$ \\
\hline 64 to 80 & $96,429(30.4)$ & $72,222(30.4)$ & 23,207 (30.4) \\
\hline $80+$ & $169,137(53.3)$ & $126,763(52.3)$ & $42,374(53.2)$ \\
\hline Female & $191,510(60.3)$ & $14,365(60.3)$ & $48,045(60.4)$ \\
\hline Unsteady gait & $216,283(68.1)$ & $162,021(68.1)$ & $54,262(68.2)$ \\
\hline Wandering & $10,165(3.2)$ & $7695(3.2)$ & $2470(3.1)$ \\
\hline Tobacco use & $27,793(8.8)$ & $20,772(8.7)$ & $7021(8.8)$ \\
\hline Fall in last 180 days & $143,666(45.2)$ & $107,588(45.2)$ & $36,074(45.3)$ \\
\hline Previous fracture in the last 180 days & $43,569(13.7)$ & $32,564(13.7)$ & $11,005(13.8)$ \\
\hline \multicolumn{4}{|l|}{ Transfer ability } \\
\hline Independent (0) & $207,607(65.4)$ & $155,698(65.4)$ & $51,909(65.2)$ \\
\hline Supervision or set up help $(1,2)$ & $43,085(13.6)$ & $32,292(13.6)$ & 10,793 (13.6) \\
\hline Limited assistance (3) & $28,550(9.0)$ & $21,244(8.9)$ & $7306(9.2)$ \\
\hline Extensive or maximal assistance $(4,5)$ & $27,608(8.7)$ & $20,750(8.7)$ & $6858(8.6)$ \\
\hline Total dependence or did not occur $(6,8)$ & $10,771(3.4)$ & $8027(3.4)$ & $2744(3.4)$ \\
\hline \multicolumn{4}{|l|}{ Primary mode of locomotion indoors } \\
\hline No aid & $117,271(36.9)$ & $88,060(37.0)$ & $29,209(36.7)$ \\
\hline Cane or walker & $158,444(49.9)$ & $118,524(49.8)$ & $39,918(50.1)$ \\
\hline Scooter, wheelchair, or did not occur & $41,911(13.2)$ & $31,427(13.2)$ & $10,483(13.2)$ \\
\hline \multicolumn{4}{|l|}{ Cognitive performance scale } \\
\hline Intact (0) & $107,057(33.7)$ & $80,222(33.7)$ & $26,833(33.7)$ \\
\hline Mild impairment $(1,2)$ & $162,604(51.2)$ & $121,648(51.1)$ & 40,954 (51.4) \\
\hline Moderate impairment $(3,4)$ & $34,339(10.8)$ & 25,883 (10.9) & 8455 (10.6) \\
\hline Severe impairment $(5,6)$ & $13,626(4.3)$ & $10,258(4.3)$ & $3368(4.2)$ \\
\hline
\end{tabular}

Risk Scale - Home Care (FRS-HC) (Fig. 3). The absolute proportion of home care recipients in each risk level who experienced a 1-year incident hip fracture ranged from 0.3 to $5.2 \%$ (Fig. 3). The odds of experiencing a fracture in each risk level as compared to the first level demonstrate a clear stepped progression, with the highest risk level having a 18.8-fold increase (95\% confidence interval, 14.6 to 24.3) (Table 3). Figure 4 demonstrates that risk level 3 had the highest (23.6\%) while risk level 6 had the lowest $(1.5 \%)$ proportion of the population. Whether the individual was ambulatory or not demonstrated the highest discriminatory power in model, followed by age and experiencing a previous fracture in the past 180 days. Other variables included were unsteady gait, cognitive impairment, transfer ability, previous falls in the past 180 days, tobacco use, wandering, locomotion ability, and sex (Fig. 2).

\section{Discrimination and predictive accuracy}

Overall, the FRS-HC demonstrated good consistency between datasets. The discriminative properties of the FRS-HC were similar between the full sample (c-statistic $=0.658)$, derivation $(c$-statistic $=0.662)$, and validation $(\mathrm{c}$-statistic $=0.645)$ datasets. Further, the absolute fracture rate for individual risk levels (Fig. 3) and the odds ratios between risk levels (Table 3) of the scale were similar between the derivation, validation, and combined datasets. 
Table 2 Univariate associations between risk factors and oneyear incident fractures for the full sample

\begin{tabular}{|c|c|}
\hline Characteristic: & $\begin{array}{l}\text { Odds ratio }(95 \% \\
\text { Confidence interval) }\end{array}$ \\
\hline Age $70+($ REF $=<70)$ & 3.5 (3.2 to 3.9$)$ \\
\hline Female (REF = female) & $1.5(1.4$ to 1.6$)$ \\
\hline Unsteady gait (REF = steady gait) & $1.6(1.5$ to 1.7$)$ \\
\hline Uses gait aid (REF = does not use gait aid) & 1.5 (1.5 to 1.6$)$ \\
\hline Wandering (REF = no wandering) & 2.5 (2.2 to 2.8$)$ \\
\hline Tobacco use (REF = no tobacco use) & $1.0(0.9$ to 1.1$)$ \\
\hline Fall in last 180 days (REF = no fall) & $1.6(1.5$ to 1.7$)$ \\
\hline $\begin{array}{l}\text { Previous fracture in the last } 180 \text { days } \\
\text { (REF = no fracture) }\end{array}$ & 1.5 (1.4 to 1.6$)$ \\
\hline Dependent in transfers (REF = independent) & 0.5 (0.4 to 0.6$)$ \\
\hline $\begin{array}{l}\text { Ambulatory indoors with or without gait } \\
\text { aid (REF = non-ambulatory) }\end{array}$ & $1.3(1.2$ to 1.4$)$ \\
\hline $\begin{array}{l}\text { Has cognitive impairment }(C P S>0) \\
\text { (REF }=\text { no cognitive impairment) }\end{array}$ & 2.2 (2.0 to 2.3 ) \\
\hline
\end{tabular}

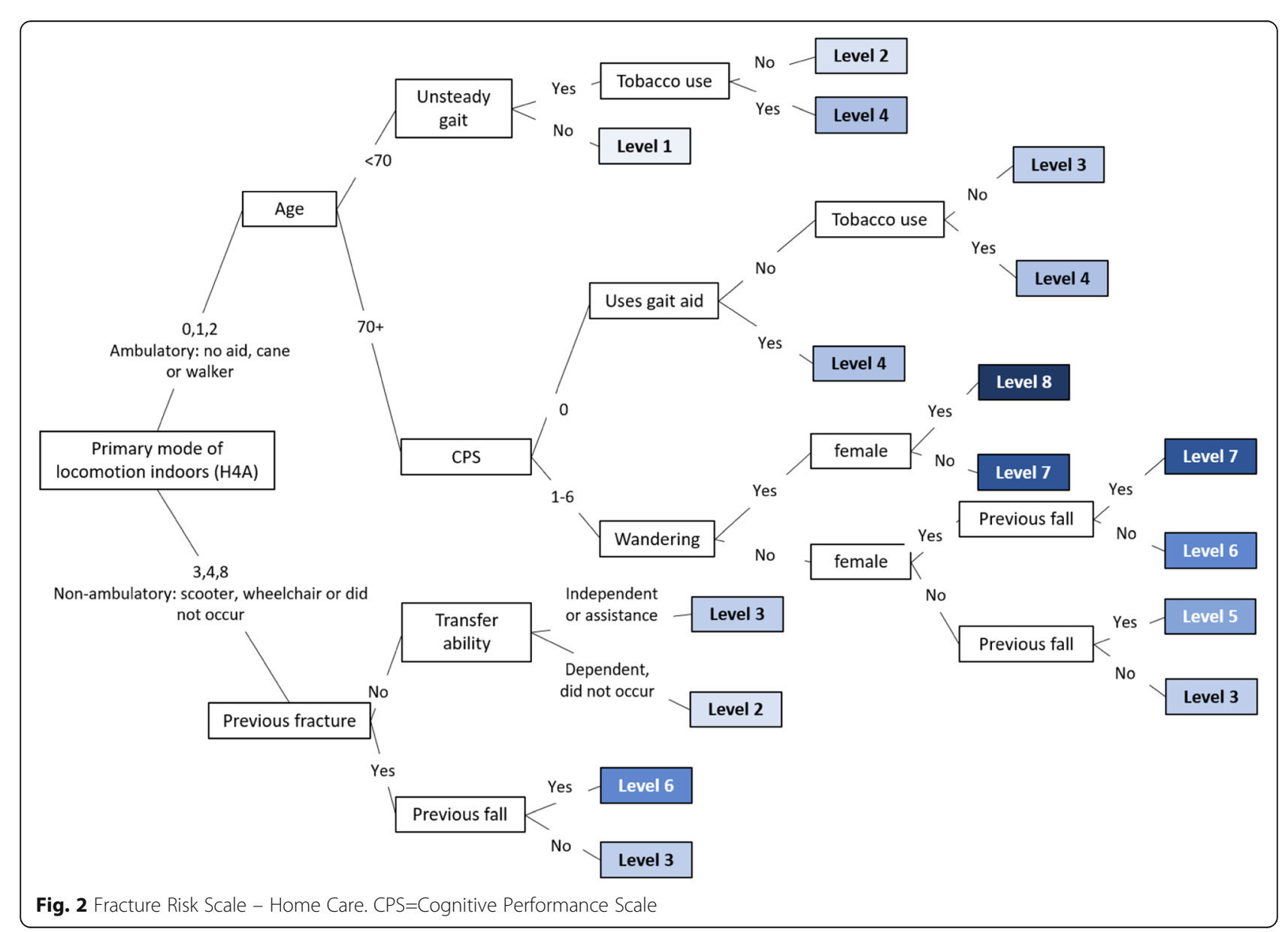

\section{Mortality rates}

After a hip fracture, 9.5\% $(N=479)$ of home care recipients died in the emergency department or as an inpatient after a mean length of stay of 20.2 days (standard deviation 38.6).

\section{Discussion}

Home care recipients have a high risk of imminent hip fracture in the next year, which can result in pain, immobility, institutionalization, and death. We developed a Fracture Risk Scale for home care (FRS-HC) which predicts one-year incident hip fracture. Our results demonstrate that the FRS-HC can both discriminate and predict home care recipients at risk for hip fracture over a one-year time period. The FRS-HC score is calculated using routinely collected home care assessment data, includes clinically relevant information for medically complex home care recipients (e.g., falls, cognitive impairment, comorbidities) and does not require additional information to be collected (e.g., bone mineral density) improving its clinical applicability and usefulness.

The FRS-HC predicts hip fracture within the next year, an important target for the vulnerable home care population. Indeed, fracture prevention strategies should

Fig. 2 Fracture Risk Scale - Home Care. CPS=Cognitive Performance Scale 


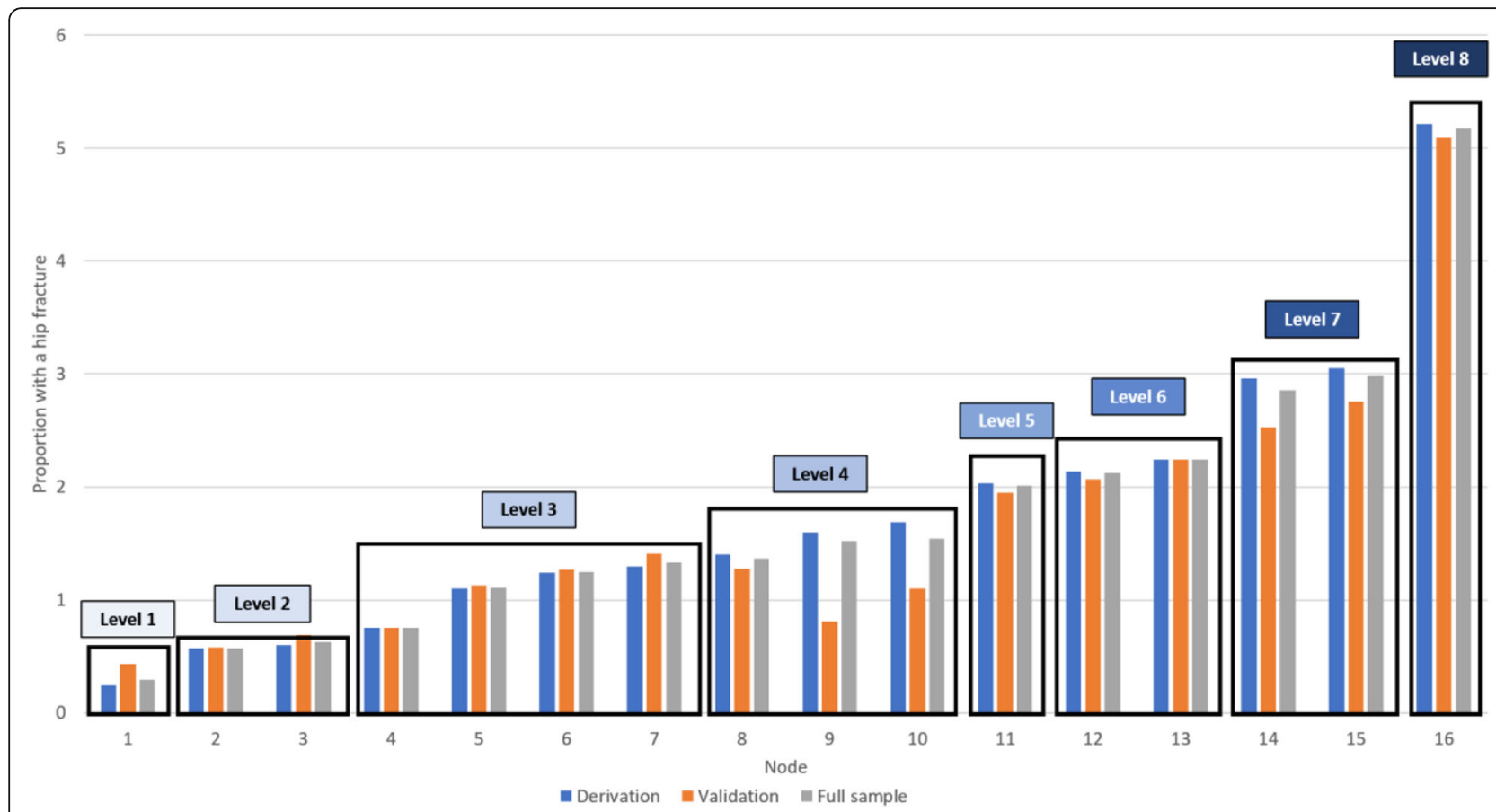

Fig. 3 Incident hip fracture rates classified by individual decision nodes and the eight risk levels for the derivation, validation, and full sample datasets

be targeted to those at imminent risk for fracture including frail, older adults with a history of previous fractures and falls like the home care population [21]. Several currently available fracture risk assessment tools can determine imminent fracture risk. For example, QFracture [18] can calculate risk for any year between one and ten, while the Garvan fracture risk assessment tool [22] calculates risk for five and ten years. FRAX currently only calculates 10-year fracture risk, though it includes consideration for the competing risk of death as age increases [23]. As such, 10-year fracture risk as calculated by FRAX decreases with age because of the increasing likelihood of death. However, as age increases risk for fracture in the next year may be underestimated which

Table 3 Odds of one-year incident hip fracture for the eight risk levels for the derivation, validation, and full sample

\begin{tabular}{|c|c|c|c|}
\hline \multirow{2}{*}{$\begin{array}{l}\text { Hip fracture } \\
\text { risk level } \\
\text { categories }\end{array}$} & Derivation sample & Validation sample & Full sample \\
\hline & OR (95\% Cl) & OR $(95 \% \mathrm{Cl})$ & OR $(95 \% \mathrm{Cl})$ \\
\hline Level 2 vs 1 & 2.4 (1.8 to 3.3$)$ & 1.4 (0.9 to 2.2 ) & 2.0 (1.6 to 2.6$)$ \\
\hline Level 3 vs 1 & 4.7 (3.5 to 6.2) & 2.7 (1.9 to 4.0$)$ & 3.9 (3.1 to 5.0$)$ \\
\hline Level 4 vs 1 & 5.7 (4.2 to 7.6$)$ & 2.8 (1.9 to 4.1$)$ & 4.6 (3.6 to 5.8 ) \\
\hline Level 5 vs 1 & 8.6 (6.4 to 11.5$)$ & 4.6 (3.1 to 6.8$)$ & 7.1 (5.6 to 8.9 ) \\
\hline Level 6 vs 1 & 9.1 (6.8 to 12.1) & 4.9 (3.4 to 7.2 ) & 7.5 (6.0 to 9.5 ) \\
\hline Level 7 vs 1 & 13.0 (9.8 to 17.3$)$ & 6.5 (4.4 to 9.4$)$ & 10.5 (8.4 to 13.2$)$ \\
\hline Level 8 vs 1 & 22.7 (16.6 to 31.2$)$ & 12.4 (7.9 to 19.2$)$ & 18.8 (14.6 to 24.3 ) \\
\hline
\end{tabular}

$O R$ odds ratio, $\mathrm{Cl}$ confidence interval could decrease the urgency for prevention strategies to be implemented [24]. The benefit of the FRS-HC is that it calculates fracture risk in the next year with data from routinely collected home care assessments thereby limiting additional documentation for an often overburdened sector.

The c-statistic of the FRS-HC is slightly lower than that of previously reported fracture risk assessment tools in other populations $[25,26]$. However, caution must be taken when comparing c-statistics across studies. The cstatistic will vary depending on the characteristics of the cohort (e.g., age range) and length of follow-up [27, 28]. Further, the c-statistic does not increase with the addition of risk factors with strong predictive values but low prevalence which may be clinically relevant and aid in determining intervention thresholds [29]. Though it may be tempting to classify all home care recipients at high risk and implement widespread fracture prevention measures, this approach is not realistic in the home care sector. Prevention resources such as staff to support exercise and nutritional counselling are often limited and expensive in the home care sector. Further, home care recipients at low risk for fracture may receive minimal benefit from preventive strategies while unnecessarily consuming resources. Indeed, prevention is more costeffective in high risk groups [30]. Targeting home care recipients who are truly at high risk will help with management of scarce resources for a sector with growing demands as the aging population increases. Though the 


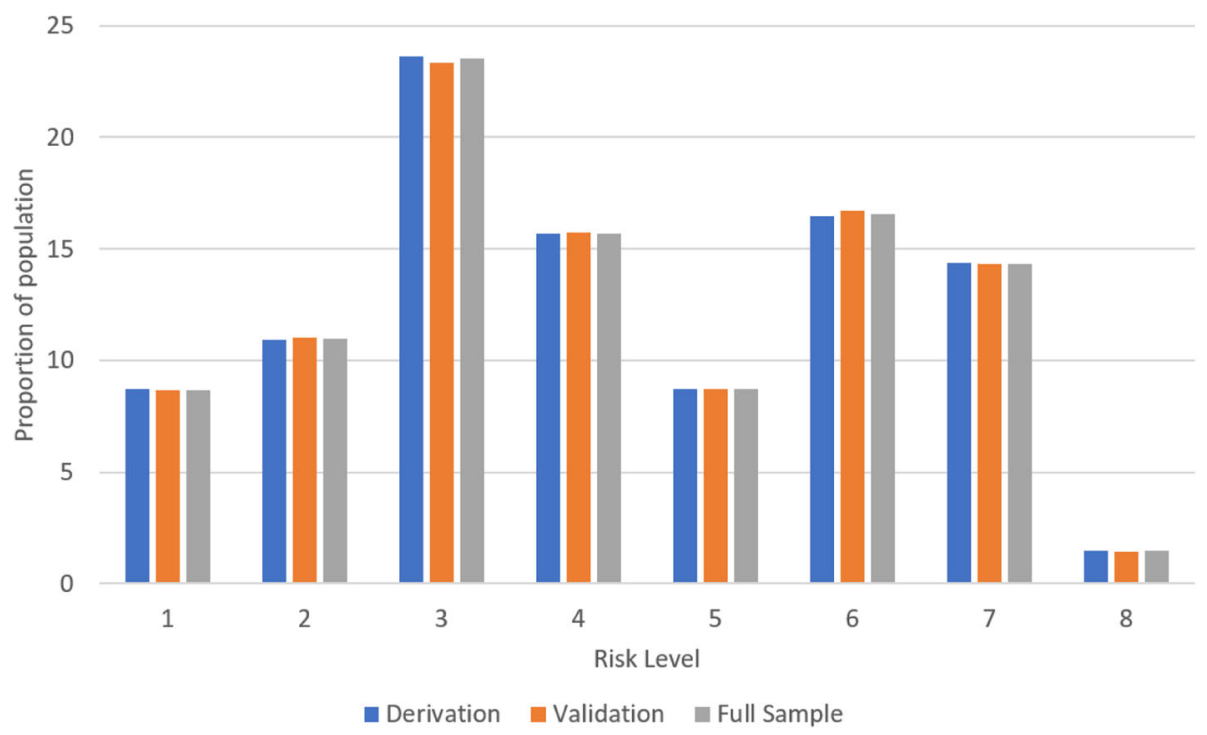

Fig. 4 Distribution of home care recipients by hip fracture risk level for the derivation, validation, and full sample datasets

c-statistic of the FRS-HC may be lower than previously developed fracture risk assessment algorithms we cannot compare across studies and regardless it will assist in allocating scare resource judiciously.

Our next step will be to develop a clinical assessment protocol (CAP) associated with the FRS-HC to link treatment and further investigation recommendations with the identified fracture risk. The CAP will classify home care recipients as low or high risk based on their FRS-HC score, and will provide evidence-based recommendations associated with their risk level. The FRS-HC identifies eight risk levels, with approximately $45 \%$ of the home care population in the lowest three risk categories [1-3] and fewer residents in the highest risk category (level 8). The lower distribution of home care recipients into higher risk categories is important as a large proportion of individuals identified as high risk can quickly overwhelm an overburdened sector, contribute to alarm fatigue, and cause many false positive identifications. Evidence-based care planning recommendations for the Fracture Risk CAP will be based on Canadian and international fracture prevention guidelines, and could include vitamin D and calcium supplementation, exercise, and pharmacological therapies [31]. The CAP will assist clinicians in identifying home care recipients at high fracture risk and recommended interventions to decrease that risk. Ultimately, the FRS-HC and associated CAP will reduce the risk of hip fracture, and healthcare costs, and improve quality of life. Given the substantial relationship between falls and fractures [32], integration of the current Falls CAP [33] and recently developed 1stFall algorithm [34], which predicts falls for home care recipients who have not previously fallen, will need to be explored. For example, it would be of value to examine the overlap between the rates that the FRS-HC and 1 stFall scales each identify people at high risk, and between recommendations in the current Falls CAP and fracture prevention recommendations.

Our study has several limitations and strengths. We were limited to the inclusion of independent variables available in the RAI-HC, and we may not have captured all relevant fracture risk factors (e.g., body mass index). Further, we were only able to capture clinical vertebral fractures which likely represents $30 \%$ of all vertebral fractures during the one-year follow-up. Thus, our estimate for incidence of vertebral fractures is likely underestimated. We did not consider bone active medications in the analysis. We chose to develop the FRS-HC using routinely collected data to facilitate ease of implementation in the home care setting, and data on medications is not immediately available to the clinicians who would use the tool. Also, similar analyses done in a large cohort of community dwelling men revealed that exclusion of individuals on bisphosphonates did not alter the findings [35]. A strength of our study is the large number of data from home care recipients that was available for developing the scale. We also included a comprehensive list of independent variables in our analyses, confirmed by an expert panel. We used linked hospital data to confirm incident hip fractures. Decision tree analysis allowed us to develop a scale with an empirically sound, visual representation of the contributing factors to hip fractures for home care recipients. Further, since decision tree analysis has no parametric assumptions, it clusters risk factors, it has a better ability to account for outliers compared with regression analysis, and it often has 
higher utility in identifying high risk individuals $[18,19]$. The FRS-HC uses items available in the RAI-HC and in the newer version of the assessment (interRAI HC). The RAI-HC and interRAI $\mathrm{HC}$ are used across several Canadian provinces and internationally, improving the usability and impact of our scale. Finally, the FRS-HC uses existing items from the RAI-HC, will automatically generate a fracture risk score based on routinely collected information, and will thereby decrease work duplication required to complete non-integrated tools such as FRAX.

\section{Conclusions}

The FRS-HC predicts hip fracture over a one-year time period, demonstrates good discriminative and predictive properties, and can be used to support care planning by identifying home care recipients at high fracture risk. Future work should compare the FRS-HC to other fracture risk assessment tools, examine the relationship between the FRS-HC and falls scales, and develop a fracture risk clinical assessment protocol to link treatment recommendations with identified fracture risk.

\section{Abbreviations}

RAI-HC: Resident Assessment Instrument - Home Care; FRS-HC: Fracture Risk Scale - Home Care; CAP: Clinical Assessment Protocol; NACRS: National Ambulatory Care Reporting System; DAD: Discharge Abstract Database

\section{Acknowledgements}

None.

\section{Authors' contributions}

CM conceptualized the study, conducted data analysis, interpreted the results, and wrote the manuscript. GI, MJ, JDA, LG, JH, and AP provided content expertise and assisted with interpretation of the results. All authors critically read, contributed to, and approved the manuscript for submission.

\section{Funding}

Caitlin MCArthur is supported by a CIHR fellowship. The funder had no role in the study, interpretation of data, or decision to submit results.

\section{Availability of data and materials}

The data analyzed in this study are not publicly available due to privacy and confidentiality restrictions pertaining to person-level health information in Canada. However, the data set creation plan and underlying analytic code are available from the corresponding author on reasonable request.

\section{Ethics approval and consent to participate}

This study was reviewed by and received ethics clearance from the Research Ethics Board at McMaster University. Necessary permissions were obtained to use the data.

\section{Consent for publication}

Not applicable.

\section{Competing interests}

None.

\section{Author details}

'McMaster University, 1200 Main Street, Hamilton, Ontario L8S 4L8, Canada. ${ }^{2}$ GERAS Centre for Aging Research, 88 Maplewood Avenue, 88 Maplewood Avenue, Hamilton, Ontario L8M 1W9, Canada. ${ }^{3}$ University of Waterloo, 200 University Avenue West, Waterloo, Ontario N2L 3G1, Canada. ${ }^{4}$ Schlegel-UW
Research Institute for Aging Research, 250 Laurelwood Drive, Waterloo, Ontario N2J OE2, Canada.

Received: 21 February 2020 Accepted: 20 July 2020

Published online: 28 July 2020

\section{References}

1. Ontario. Home and Community Care. Available from: https://www.ontario. ca/page/homecare-seniors. [cited 2019 Jan 11].

2. Campitelli MA, Bronskill SE, Hogan DB, Diong C, Amuah JE, Gill S, et al. The prevalence and health consequences of frailty in a population-based older home care cohort: a comparison of different measures. BMC Geriatr. 2016; 16(1):133 016-0309-z.

3. Stolee P, Poss J, Cook RJ, Byrne K, Hirdes JP. Risk factors for hip fracture in older home care clients. Journals Gerontol A. Biol Sci Med Sci. 2009;64(3): 403-10.

4. Sugarman JR, Connell FA, Hansen A, Helgerson SD, Jessup MC, Lee H. Hip fracture incidence in nursing home residents and community-dwelling older people, Washington state, 1993-1995. J Am Geriatr Soc. 2002;50(10): 1638-43.

5. Jaglal SB, Sherry PG, Schatzker J. The impact and consequences of hip fracture in Ontario. Can J surgery J Can Chir. 1996;39(2):105-11.

6. Katelaris AG, Cumming RG. Health status before and mortality after hip fracture. Am J Public Health. 1996;86(4):557-60.

7. Hopkins RB, Burke N, Von Keyserlingk C, Leslie WD, Morin SN, Adachi JD, et al. The current economic burden of illness of osteoporosis in Canada. Osteoporos Int. 2016;27(10):3023-32 Available from: http://www.ncbi.nlm. nih.gov/pubmed/27166680. [cited 2018 Jan 22].

8. Kanis JA, Johnell O, Oden A, Johansson H, McCloskey E. FRAX and the assessment of fracture probability in men and women from the UK. Osteoporos Int. 2008;19(4):385-97.

9. Morris JN, Fries BE, Steel K, Ikegami N, Bernabei R, Carpenter Gl, et al. Comprehensive clinical assessment in community setting: applicability of the MDS-HC. J Am Geriatr Soc. 1997;45(8):1017-24 Available from: http://www.ncbi.nlm.nih.gov/pubmed/9256857. [cited 2018 Feb 24].

10. loannidis G, Jantzi M, Bucek J, Adachi JD, Giangregorio L, Hirdes J, et al. Development and validation of the Fracture Risk Scale (FRS) that predicts fracture over a 1-year time period in institutionalised frail older people living in Canada: an electronic record-linked longitudinal cohort study. BMJ Open. 2017;7(9):e016477 Available from: http://www.ncbi.nlm.nih.gov/ pubmed/28864698. [cited 2018 Jan 16].

11. McArthur C, loannidis G, Jantzi M, Hillier L, Adachi JD, Giangregorio L, et al. A Higher Proportion of Home Care Recipients Experience Nonhip Fractures Than Long-Term Care Residents. J Am Med Dir Assoc. 2019; Available from: http://www.ncbi.n/m.nih.gov/pubmed/31866418. [cited 2020 Jan 17].

12. McArthur C, loannidis G, Jantzi M, Adachi JD, Giangregorio L, Hirdes J, et al. Are They Really a Different Population? Comparing Fracture Risk Factors Between Home Care Recipients and Long-Term Care Residents. J Bone Miner Res. 2018;32(suppl 1).

13. Haaland DA, Cohen DR, Kennedy CC, Khalidi NA, Adachi JD, Papaioannou A. Closing the osteoporosis care gap: increased osteoporosis awareness among geriatrics and rehabilitation teams. BMC Geriatr. 2009;9:28 Available from: http://www.ncbi.nlm.nih.gov/pubmed/19602246. [cited 2020 Jan 17].

14. Hirdes JP, Ljunggren G, Morris JN, Frijters DHM, Finne Soveri H, Gray L, et al. Reliability of the interRAI suite of assessment instruments: a 12-country study of an integrated health information system. BMC Health Serv Res. 2008;8(1):277. [cited 2018 Feb 24]. https://doi.org/10.1186/1472-6963-8-277.

15. ClHI. Data Quality Documentation, Discharge Abstract Database - Multi-Year Information. 2012

16. $\mathrm{CIHI}$. Data Quality Documentation National Ambulatory Care Reporting System - Multi-Year Information. 2012.

17. O'Donnell S, Canadian Chronic Disease Surveillance System (CCDSS) Osteoporosis Working Group. Use of administrative data for national surveillance of osteoporosis and related fractures in Canada: results from a feasibility study. Arch Osteoporos. 2013:8(1-2):143 Available from: http://www.ncbi.nlm.nih.gov/pubmed/23740086. [cited 2018 Aug 23].

18. Hippisley-Cox J, Coupland C. Predicting risk of osteoporotic fracture in men and women in England and Wales: prospective derivation and validation of QFractureScores. BMJ. 2009:339(nov19 1):b422. [cited 2018 Feb 24]. https://doi.org/10.1136/bmj.b4229. 
19. Chen JS, Sambrook PN, Simpson JM, March LM, Cumming RG, Seibel MJ, et al. A selection strategy was developed for fracture reduction programs in frail older people. J Clin Epidemiol. 2010;63(6):679-85 Available from: https://inkinghub.elsevier.com/retrieve/pii/S0895435609002716. [cited 2019 Dec 13].

20. Kass GV. An Exploratory Technique for Investigating Large Quantities of Categorical Data. Appl Stat. 1980;29(2):119. [cited 2018 May 8]. https://doi.org/10.2307/2986296?origin=crossref.

21. Roux C, Briot K. Imminent fracture risk. Vol. 28, Osteoporosis International. London: Springer; 2017. p. 1765-9.

22. Nguyen ND, Frost SA, Center JR, Eisman JA, Nguyen TV. Development of a nomogram for individualizing hip fracture risk in men and women. Osteoporos Int. 2007;18(8):1109-17.

23. Kanis JA, Harvey NC, Johansson H, Odén A, McCloskey EV, Leslie WD. Overview of fracture prediction tools. J Clin Densitom. 2017;20(3):444-50.

24. Bolland M, Jackson R, Gamble G, Bmj AG-, 2013 undefined. Discrepancies in predicted fracture risk in elderly people. bmj.com [Internet]. 2013 [cited 2020 May 26]; Available from: http://www.bmj.com/permissionsSubscribe : http://www.bmj.com/subscribe, http://www.bmj.com/.

25. Reber KC, König HH, Becker C, Rapp K, Büchele G, Mächler S, et al. Development of a risk assessment tool for osteoporotic fracture prevention: A claims data approach. Bone. 2018:110:170-6.

26. Rubin KH, Abrahamsen B, Friis-Holmberg T, Hjelmborg JVB, Bech M, Hermann AP, et al. Comparison of different screening tools (FRAX, OST, ORAI, OSIRIS, SCORE and age alone) to identify women with increased risk of fracture. A population-based prospective study. Bone. 2013;56(1):16-22.

27. Kanis JA, Oden $\mathrm{A}$, Johansson $\mathrm{H}$, et al. Expressing fracture risk. Osteoporos Int. 2008;19:593-4.

28. Kanis JA, Oden A, Johnell O, Johansson H, De Laet C, Brown J, et al. The use of clinical risk factors enhances the performance of BMD in the prediction of hip and osteoporotic fractures in men and women. Osteoporos Int. 2007; 18(8):1033-46

29. Kanis JA, Oden A, Johansson $\mathrm{H}$, et al. Pitfalls in the external validation of FRAX. Osteoporos Int. 2012;23:423-31.

30. Gillespie LD, Robertson MC, Gillespie WJ, Sherrington C, Gates S, Clemson $L M$, et al. Interventions for preventing falls in older people living in the community. Cochrane database Syst Rev. 2012;(9):CD007146. https:/doi.org/ 10.1002/14651858.CD007146.pub3.

31. Papaioannou A, Morin S, Cheung AM, Atkinson S, Brown JP, Feldman S, et al. 2010 clinical practice guidelines for the diagnosis and management of osteoporosis in Canada: summary. CMAJ. 2010;182(17):1864-73 Available from: http://www.scopus.com/inward/record.url?eid=2-s2.0-7864968081 7\&partnerl $\mathrm{D}=40 \& \mathrm{md} 5=111 \mathrm{be} 0 \mathrm{ae} 7922 \mathrm{fd} 5 \mathrm{a} 72316 \mathrm{ca} 8 \mathrm{~b} 160 \mathrm{~cd} 7 \mathrm{c}$.

32. Burns $E R$, Stevens JA, Lee R. The direct costs of fatal and non-fatal falls among older adults - United States. J Safety Res. 2016;58:99-103 Available from: https://linkinghub.elsevier.com/retrieve/pii/S0022437516300172. [cited 2019 Nov 8]

33. Morris JN, Berg K, Bjorkgren M, Finne-Soveri H, Fries BE, Frijters D, et al. interRAI Clinical Assessment Protocols (CAPs) for Use with Community and Long-Term Care Assessment Instruments. Version 9.1. Washington, DC interRAl; 2010

34. Kuspinar A, Hirdes JP, Berg K, McArthur C, Morris JN. Development and validation of an algorithm to assess risk of first-time falling among home care clients. BMC Geriatr. 2019;19(1):264. [cited 2019 Dec 13]. https://doi.org/ 10.1186/s12877-019-1300-2.

35. Su Y, Kwok TCY, Cummings SR, Yip BHK, Cawthon PM. Can classification and regression tree analysis help identify clinically meaningful risk groups for hip fracture prediction in older American men (the MrOS cohort study)? JBMR Plus. 2019;3(10):e10207.

\section{Publisher's Note}

Springer Nature remains neutral with regard to jurisdictional claims in published maps and institutional affiliations.

Ready to submit your research? Choose BMC and benefit from:

- fast, convenient online submission

- thorough peer review by experienced researchers in your field

- rapid publication on acceptance

- support for research data, including large and complex data types

- gold Open Access which fosters wider collaboration and increased citations

- maximum visibility for your research: over $100 \mathrm{M}$ website views per year

At $\mathrm{BMC}$, research is always in progress.

Learn more biomedcentral.com/submissions 